\title{
Investigation of Different MicroCT Scanner Configurations by GEANT4 Simulations
}

\author{
Maryam Khodaverdi, Arion F. Chatziioannou, Member, IEEE, Simone Weber, Karl Ziemons, Member, IEEE, \\ Horst Halling, and Uwe Pietrzyk, Member, IEEE
}

\begin{abstract}
This study has been performed to design the combination of the new ClearPET (ClearPET is a trademark of the Crystal Clear Collaboration), a small animal positron emission tomography (PET) system, with a micro-computed tomography (microCT) scanner. The properties of different microCT systems have been determined by simulations based on GEANT4. We will demonstrate the influence of the detector material and the $\mathrm{X}$-ray spectrum on the obtained contrast. Four different detector materials (selenium, cadmium zinc telluride, cesium iodide and gadolinium oxysulfide) and two $\mathrm{X}$-ray spectra (a molybdenum and a tungsten source) have been considered. The spectra have also been modified by aluminum filters of varying thickness. The contrast between different tissue types (water, air, brain, bone and fat) has been simulated by using a suitable phantom. The results indicate the possibility to improve the image contrast in microCT by an optimized combination of the $\mathrm{X}$-ray source and detector material.
\end{abstract}

Index Terms-Cadmium Zinc Telluride (CZT), Cesium Iodide (CsI), ClearPET, contrast, Gadolinium Oxysulfide (GdOS), GEANT4, Selenium (Se), X-ray detectors, X-ray tomography.

\section{INTRODUCTION}

$\mathbf{X}$ -RAY micro-computed tomography (microCT) is a powerful nondestructive technique, which provides high resolution images of the internal structure of samples. Its ability to provide detailed anatomical information in small animal research has made microCT a very popular instrument. It has gained increasing importance employed in combination with other tomographic devices like positron emission tomography (PET) [1], [2] or single photon emission computed tomography (SPECT) [3], [4]. We are planning to combine the new ClearPET ${ }^{1}$ Neuro [5]-[8], which is currently being developed in the Crystal Clear Collaboration (CCC), with a microCT scanner in a single system. Apart from the direct link of the

Manuscript received November 14, 2003. This work was supported in part by the German Academic Exchange Service (DAAD).

M. Khodaverdi is with Central Institute for Electronics, Forschungszentrum Jülich GmbH, 52425 Jülich, Germany and also with the Department of Physics, University of Wuppertal, 42119 Wuppertal, Germany (e-mail: m.khodaverdi@fz-juelich.de).

A. F. Chatziioannou is with Crump Institute for Molecular Imaging, University of California Los Angeles, Los Angeles, CA 90095 USA (e-mail: archatziioann@mednet.ucla.edu).

S. Weber, K. Ziemons, and H. Halling are with the Central Institute for Electronics, Forschungszentrum Jülich GmbH, 52425 Jülich, Germany (e-mail: s.weber@fz-juelich.de; k.ziemons@fz-juelich.de; h.halling@fz-juelich.de).

U. Pietrzyk is with Institute of Medicine, Forschungszentrum Jülich GmbH, 52425 Jülich, Germany and the Department of Physics, University of Wuppertal, 42119 Wuppertal, Germany (e-mail: u.pietrzyk@fz-juelich.de).

Digital Object Identifier 10.1109/TNS.2004.843098

${ }^{1}$ ClearPET is a trademark of the Crystal Clear Collaboration (CCC).
TABLE I

Properties OF THE USED X-RAY CONVERTERS

\begin{tabular}{|l|c|c|c|c|}
\hline & Se & CsI & CZT & GdOS \\
\hline thickness & $250 \mu \mathrm{m}$ & $500 \mu \mathrm{m}$ & $2 \mathrm{~mm}$ & $45 \mu \mathrm{m}$ \\
\hline pixel size & $66 \mu \mathrm{m}$ & $100 \mu \mathrm{m}$ & $2 \mathrm{~mm}$ & $50 \mu \mathrm{m}$ \\
\hline
\end{tabular}

anatomical data to the physiological data from PET, there will be the option to implement a CT-based attenuation correction. Using a commercially available microCT scanner for this application would require significant modification of the CT-scanner and possibly restrict the design of the PET system, so that the construction of a suitable microCT scanner for the PET system seems to be the more appropriate solution. In preparation for such an instrument we will demonstrate simulations of different microCT configurations. Particular emphasis will be placed on the achievable contrast, which is of crucial importance because of the small size of the scanned object.

\section{Simulation SETUP}

A CT simulation has been implemented using the GEANT4 Monte Carlo simulation package which is the latest version of the detector description and simulation tool developed at CERN, Geneva, Switzerland [9]. The simulation has been designed to describe the image formation in CT starting from the generation of the X-ray photons up to the detection of these photons by the absorption in the sensitive detector. The $\mathrm{X}$-ray source is modeled by a randomly distributed set of photons whose energy distribution is adjusted to resemble that of a measured spectrum. Within the GEANT4 framework the relevant interaction processes for X-rays in matter, the photoelectric effect and the Compton scattering are simulated while tracking the path of the photons. Summed over all simulated photons, the energy deposited in each pixel element of the detector is used to construct the projection image.

In order to simulate different CT-scanners various detector materials and X-ray source spectra with different aluminum filtration from existing systems have been chosen. As X-ray converters, amorphous selenium (Se) [2], Cesium Iodide (CsI), Cadmium Zinc Telluride (CZT), and Gadolinium Oxysulfide (GdOS) [10], [11] have been taken into consideration. The characteristics of the detectors are listed in Table I. For comparison also an ideal detector, which detects all incident photons has been used.

Fig. 1 shows the spectra of the X-ray sources with a tungsten and a molybdenum anode, which have been obtained under similar conditions by measurements with single pixel CZT detectors [2], [12]. In order to analyze the effect of filtering we 

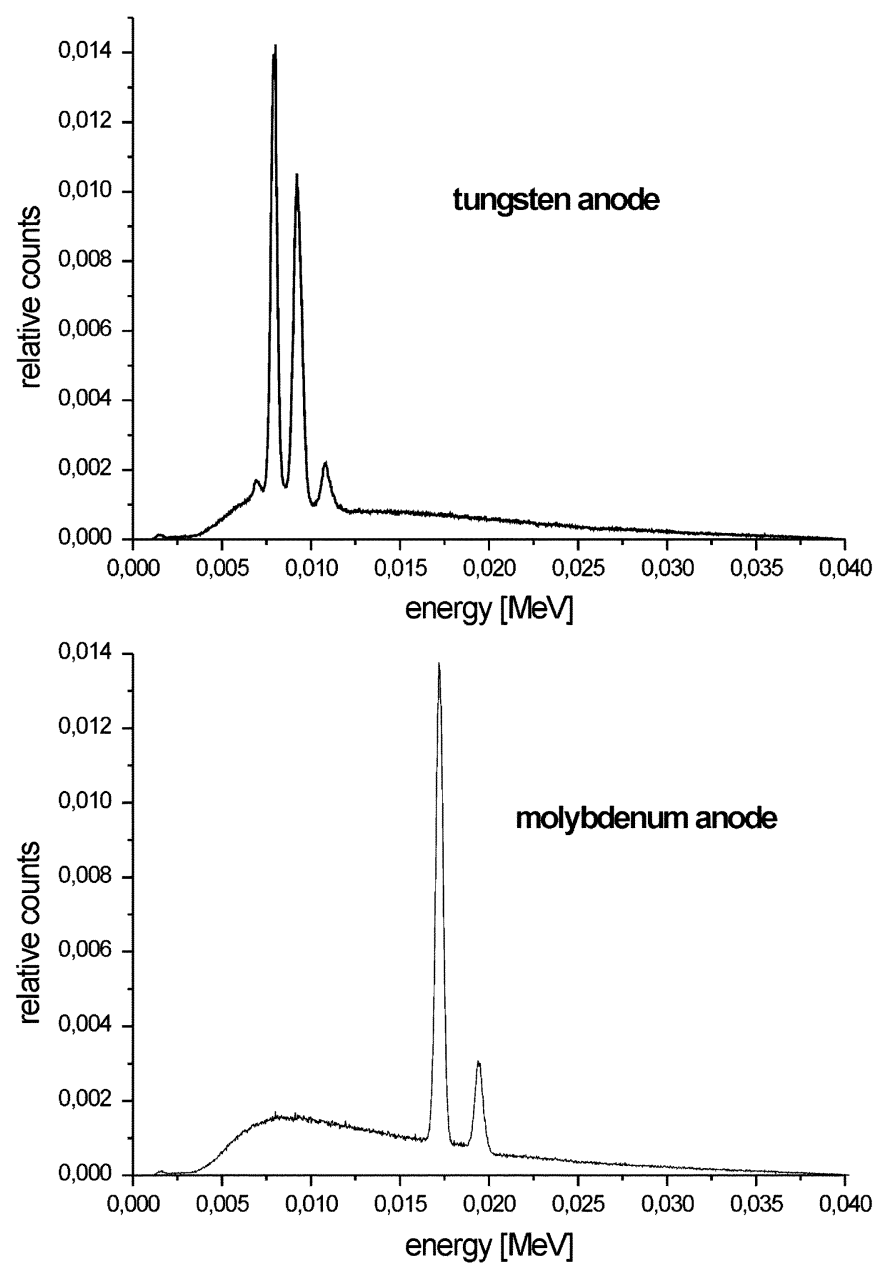

Fig. 1. Spectra of the used X-ray sources have been measured with a $2 \times 2 \times$ $2 \mathrm{~mm}^{3}$ CZT pixel detector by [2], [8].

added aluminum filters of different thickness $(0.25,0.5,0.75$, and $1 \mathrm{~mm}$ ) to the simulation. For that purpose the filter effect on both measured spectra was simulated and the resulting filtered spectra were used as input data for later CT simulations. This enables us to keep the number of the generated photons equal to those passing the phantom because the filtration has already been fulfilled. Furthermore, less photons need to be generated which leads to a shorter computing time. Since all listed components exist and are currently used in radiology, the composed systems can be assumed as realistic.

For the determination of the contrast between tissues relevant for small animal measurements, we designed a mathematical phantom consisting of water, bone, brain, fat, and air (Fig. 2). Four $2 \mathrm{~cm}$ long cuboids of different tissue types are arranged in a bigger cuboid of the same length filled with water. The different material properties have been taken from the NIST (National Institute of Standards and Technology) database (Table II). The geometry of the simulated systems are sketched in Fig. 3. The planar detector is placed $28 \mathrm{~cm}$ away from the point source, while the photons are randomly generated within a defined solid angle.

Combining the listed detector materials, sources and filters 44 different scanner configurations have been simulated. For each projection $10^{8}$ photon were generated, whereas up to 3 min are needed for $10^{6}$ photons on a pc (PentiumIII, $700 \mathrm{MHz}$ ). In order

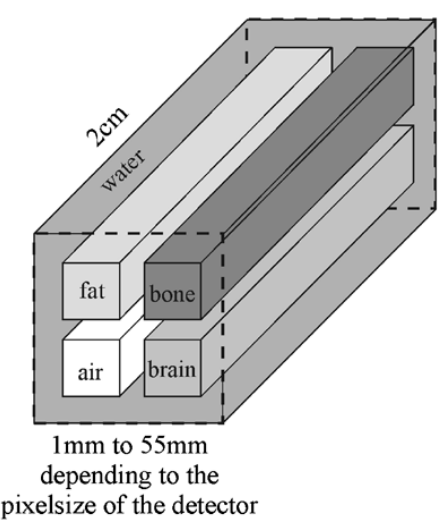

Fig. 2. Phantom consists of a water box containing smaller volumes made of different tissue types (fat, air, brain, and bone).

TABLE II

PROPERTIES OF THE DifFERENT TISSUE TYPES OCCURRING IN THE PHANTOM

\begin{tabular}{|l|l|l|}
\hline material & density $\left[\mathrm{g} / \mathrm{cm}^{3}\right]$ & composition \\
\hline Air & $1.205 \times 10^{-3}$ & $\mathrm{~N}_{0.75}, \mathrm{O}_{0.25}$ \\
\hline Water & 1.0 & $\mathrm{H}_{2}, \mathrm{O}_{1}$ \\
\hline Bone & 1.92 & $\begin{array}{l}\mathrm{H}_{0.034}, \mathrm{C}_{0.155}, \mathrm{~N}_{0.042}, \mathrm{O}_{0.435}, \mathrm{Mg}_{0.002}, \\
\mathrm{P}_{0.103}, \mathrm{~S}_{0.003}, \mathrm{Ca}_{0.225}\end{array}$ \\
\hline Brain & 1.04 & $\begin{array}{l}\mathrm{H}_{0.107}, \mathrm{C}_{0.145}, \mathrm{~N}_{0.022}, \mathrm{O}_{0.712}, \mathrm{P}_{0.004}, \\
\mathrm{~S}_{0.002}, \mathrm{Cl}_{0.003}, \mathrm{~K}_{0.003}\end{array}$ \\
\hline Fat & 0.95 & $\begin{array}{l}\mathrm{H}_{0.114}, \mathrm{C}_{0.598}, \mathrm{~N}_{0.007}, \mathrm{O}_{0.278}, \mathrm{Na}_{0.001}, \\
\mathrm{~S}_{0.001}, \mathrm{Cl}_{0.001}\end{array}$ \\
\hline
\end{tabular}

to save computing time only projections have been analyzed to determine the contrast between the materials. To demonstrate that the results obtained from the projections can be transferred to tomographic images, also complete CT simulations have been computed in selected cases.

The compositions Mo-GdOS, Mo-CZT, W-GdOS, and W-CZT have been chosen for the tomography simulations. 180 projections with about 21000 photons per pixel per projection have been generated. Since the filtration does usually not change the relative order of the obtained contrast curves and the low energy photons are mainly absorbed, only spectra with $1 \mathrm{~mm}$ filtration were taken for the CT simulation. The projections have been reconstructed with a filtered back projection algorithm.

\section{RESULTS}

\section{A. Simulated Spectra}

In order to get the filtered spectra of the tungsten and molybdenum source $10^{6}$ photons have been generated and attenuated by aluminum plates of different thickness. The spectra obtained behind the filters with an ideal detector are shown in Fig. 4. It is obvious that for both tungsten and molybdenum the photons with lower energy are absorbed by the aluminum filters. However, due to higher energy of the strong characteristic peak of the molybdenum spectrum, the change in spectrum is less drastic. These spectra have been used as input data for all further simulations.

\section{B. Contrast in Projections}

Within the simulated projections different regions of interest (ROI) based on the image of the tissue types can be defined 


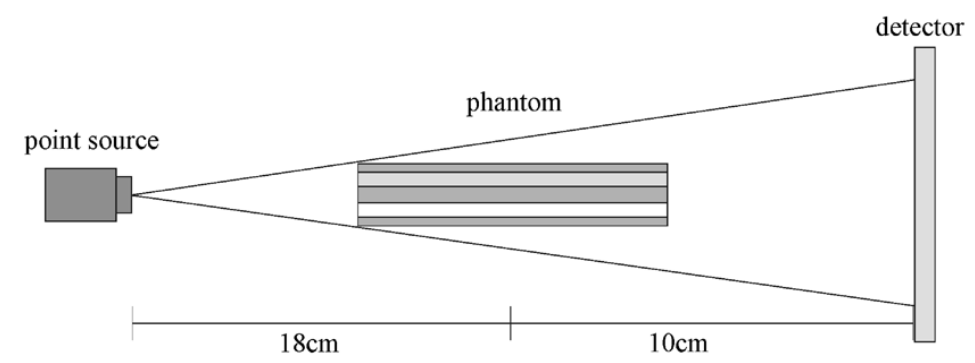

Fig. 3. Geometry of the simulated setups shows the position of the components.
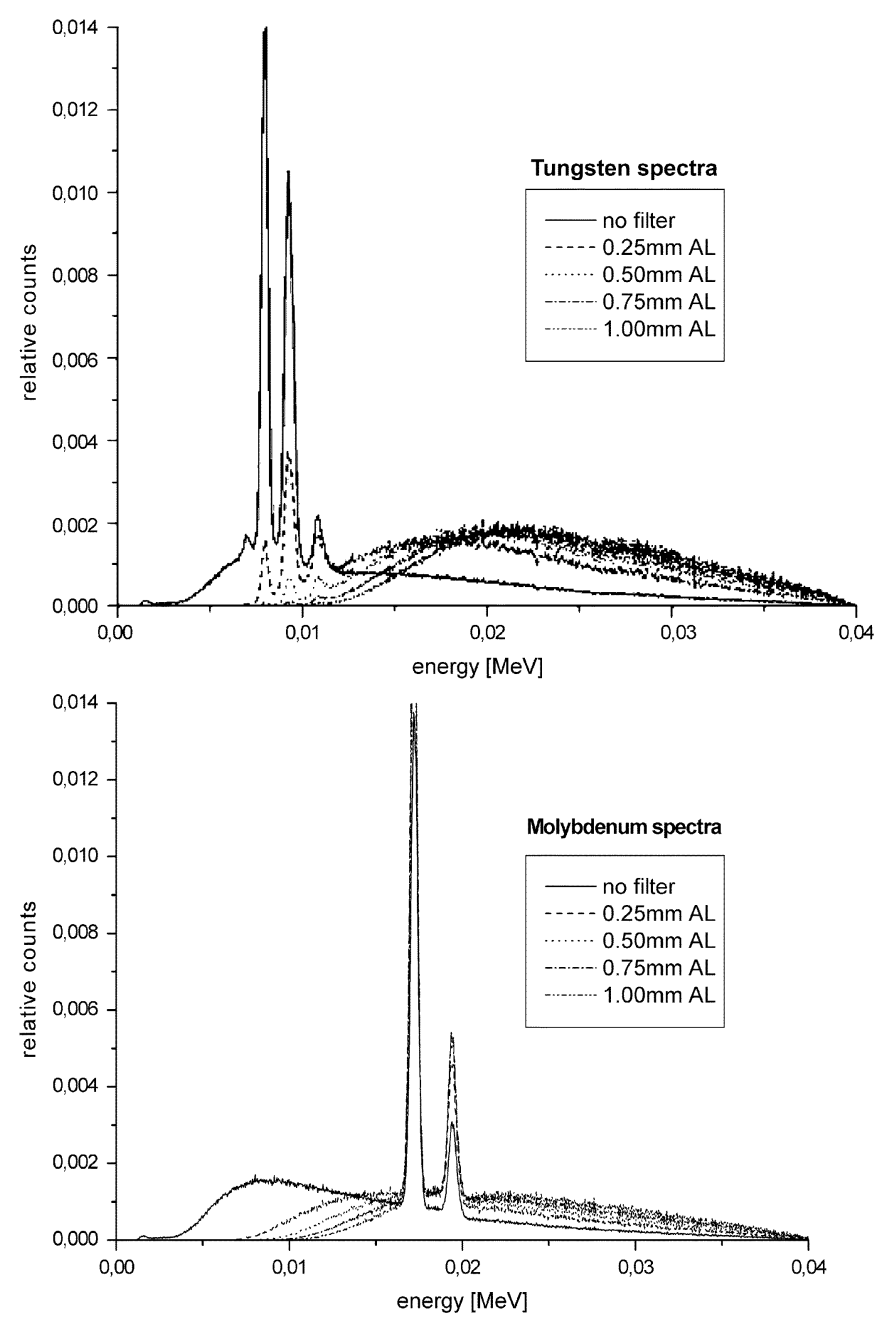

Fig. 4. Measured spectra have been filtered by simulation and used as input data for further simulations.

(Fig. 5). The mean value of the intensities $I_{\text {tissue }}$ in each ROI can be used to analyze the projection in terms of contrast. The contrast between two different tissue types is calculated by

$$
\text { contrast }=\frac{I_{\text {tissue } 1}-I_{\text {tissue2 }}}{I_{\text {tissue1 }}+I_{\text {tissue2 }}} \text {. }
$$

The obtained values depending on filtration with Aluminum (Al-filter) are shown in Fig. 6, in which the contrast curves for the Molybdenum spectrum (Mo-spectrum) and Tungsten spectrum (W-spectrum) are separated due to better comparison. Since the contrast between bone and other materials is in principle high, only the diagram for water-bone is shown here as an

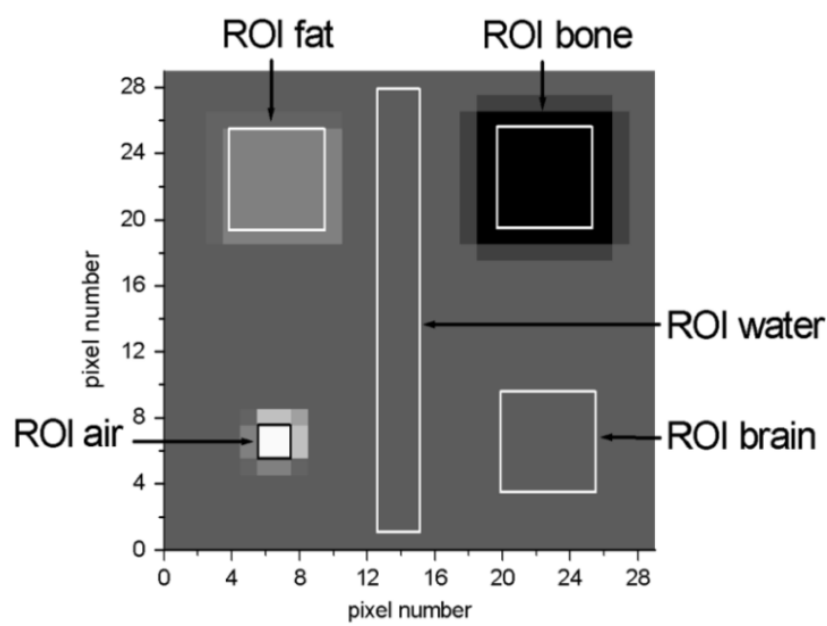

Fig. 5. Different ROI are exemplary shown here in one simulated projection. The ROI air is meant as a reference.

example. However, the contrast between soft tissues as water, fat and brain is quite low. Fig. 7 gives an idea of the reason for this phenomenon which is based on the difference between the attenuation coefficients of different materials. While the attenuation curves of the soft materials are relatively close to each other, the attenuation curve for bone is clearly higher. The fact that the difference in attenuation becomes smaller for higher energies reflects the decrease of all contrast curves with increasing filtration. Besides, the smaller the mean contrast value the higher the fluctuation within the curves. This can be explained by the increasing relative error.

Comparing the calculated contrast between all possible tissue combinations and scanner configurations one can observe significant variation. Especially, the combination of Mo $\mathrm{X}$-ray source and GdOS detector seems to outperform all other detector/X-ray source configurations. The reason for this could be the relatively high efficiency of this detector material compared to the others. In contrast to this system the combination between $\mathrm{W}$ source and CZT seems to provide the lowest contrast values. Furthermore, arrangements of configuration groups appear in the diagrams. Except for water-bone the configurations with a Mo source tend to yield higher contrast values than with a tungsten source, which can be explained by the low mean energy in the Mo spectrum. The simulations with ideal detector material produce lower contrast value compared to those detectors simulated with the same source type. This is because an ideal detector has the same quantum efficiency at all energies, so that also the photons with higher energy are 


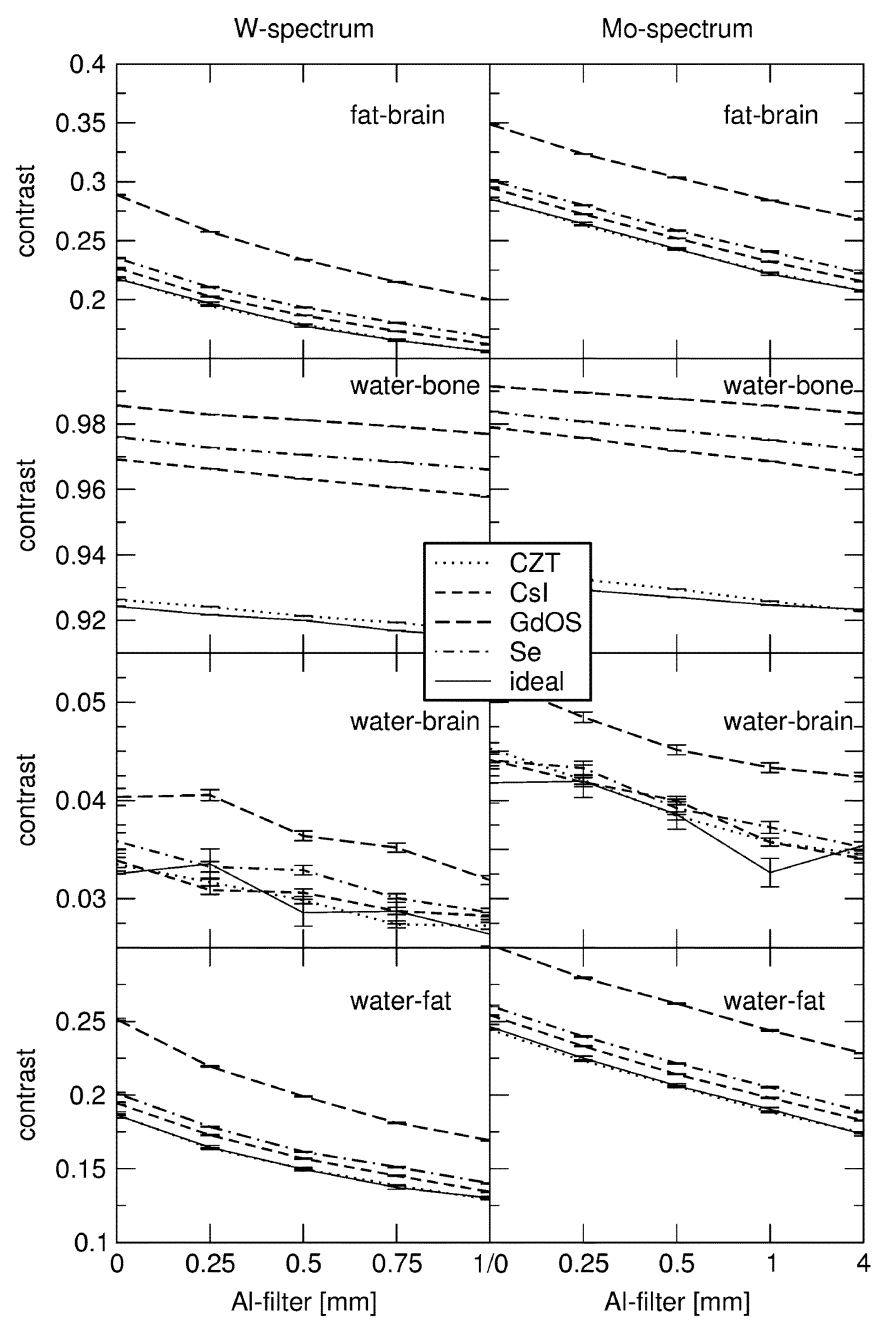

Fig. 6. Contrast curves (1) between the different tissue types water, fat, brain, and bone. The indicated errors were obtained by taking the limited statistics into account.

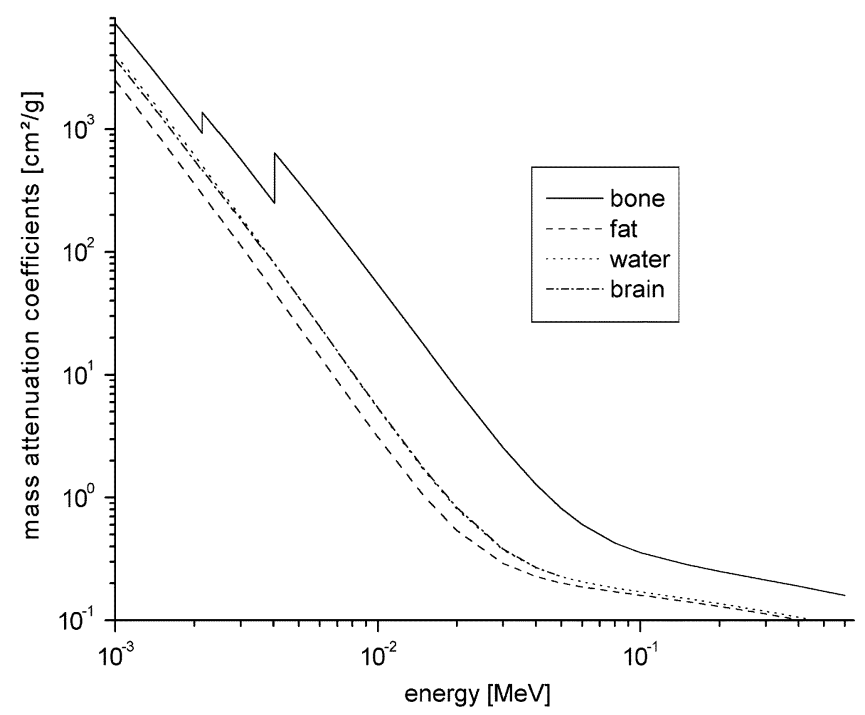

Fig. 7. Attenuation curves of water fat and brain are close whereas the curve for bone is visibly higher.

totally absorbed although they don't noticeably contribute to the contrast.
TABLE III

CONTRAST BETWEen THE TISSUE TYPES IN THE CT SimULATION

\begin{tabular}{|l|c|c|c|c|}
\hline & Water-bone & Water-fat & Water-brain & Brain-fat \\
\hline Mo-GdOS & 0,55 & 0,070 & 0,018 & 0,088 \\
\hline Mo-CZT & 0,53 & 0,065 & 0,018 & 0,083 \\
\hline W-GdOS & 0,52 & 0,064 & 0,018 & 0,082 \\
\hline W-CZT & 0,50 & 0,065 & 0,016 & 0,081 \\
\hline
\end{tabular}

\section{Contrast in CT Images}

Table III shows the contrast obtained in the reconstructed images of the phantom using the calculated projections as input. The intensities used for this calculation have been obtained by averaging 20 volume elements which could be identified with the corresponding tissue type. Even though the limited statistics does not allow a comparison as detailed as in the projections shown before, the CT simulations clearly confirm that the configuration Mo-GdOS yields the highest and W-CZT the lowest contrast values. Consequently, knowing the contrast in projections one can qualitatively deduce the values in a $\mathrm{CT}$ image.

\section{CONCLUSION}

By computer simulations of different realistic microCT setups we investigated the influence of choice of the source-detector combination on the system performance. The intention was to optimize the performance such that the contrast between different tissue types becomes maximal.

The results can be summarized by the observation that the main source for contrast in microCT will be the low energy contribution of the radiation. This is due to the small sample sizes which will not lead to sufficient attenuation of higher energy radiation. Hence, the use of an X-ray source with a relatively low mean energy like molybdenum is advantageous. At the same time the very same argument can be applied for the choice of the detector. The results for an ideal detector demonstrate that the material with higher detection efficiency does not necessarily provide the highest contrast. Yet, a relatively higher detection rate at low energies must be achieved.

Concerning the design of ClearPET Neuro, which is under development, the integration of a CT in the future has been taken into consideration. Since GdOS shows a high absorption behavior for both relevant target materials, it has turned out to be the scintillation material of choice. For applications with small animals like mice and rats an X-ray tube with a Molybdenum target is suitable. For planed applications with small monkeys the probably increasing low energy radiation exposure compared to using a Tungsten target should be taken into consideration.

\section{ACKNOWLEDGMENT}

The authors would like to thank P. L. Chow and A. L. Goertzen for fruitful discussions and the provision of the X-ray spectra. Also, they offer many thanks to F. Rannou for the patience and help at the beginning of this study. 


\section{REFERENCES}

[1] P. L. Chow, R. Rannou, and A. F. Chaziioannou, "Attenuation correction for a 3D small animal PET tomograph, using X-ray microCT," Mol. Imag. Biol., vol. 4, no. 4, p. S17, 2002.

[2] A. L. Goertzen, "Development of a Combined microPET and Microct System for Mouse Imaging," Ph.D. dissertation, University of California at Los Angeles, 2003.

[3] K. Iwata, M. C. Wu, and B. H. Hasegawa, "Design of combined X-ray CT and SPECT systems for small animals," in Proc. IEEE Nuclear Science Symp. IEEE Nuclear Science Symp. and Medical Imaging Conf., vol. 3, Seattle, WA, Oct. 24-30, 1999, pp. 1608-1612.

[4] G. A. Kastis, L. R. Furenlid, and D. W. Wilson, "Compact CT/SPECT small-animal imaging system," in Proc. IEEE Nuclear Science Symp. and Medical Imaging Conf., Norfolk, VA, Nov. 10-16, 2002.

[5] M. Streun et al., "A PET system with free running ADC's," Nucl. Instr. Meth., vol. 486, no. 1-2, pp. 18-21, Jun. 2002.

[6] M. Streun et al., "Coincidence detection by digital processing of freerunning sampled pulses," Nucl. Instr. Meth., vol. 487, no. 3, pp. 530-534, Jul. 2002.
[7] M. Streun et al., "Pulse shape discrimination of LSO and LuYAP scintillators for depth of interaction detection in PET," IEEE Trans. Nucl. Sci., vol. 50, no. 3, pp. 344-347, Jun. 2003.

[8] U. Heinrichs, "Design optimization of the PMT-Clear-PET prototypes based on simulation studies with GEANT3," IEEE Trans. Nucl. Sci., pt. 2, vol. 50, no. 5, pp. 1428-1432, Oct. 2003.

[9] S. Agostinelli et al., "GEANT4-A simulation toolkit," Nucl. Instr. Meth., vol. A 506, pp. 250-303, 2003.

[10] M. J. Paulus, H. Sari-Sarraf, and S. S. Gleason, "A new X-ray computed tomography system for laboratory mouse imaging," IEEE Trans. Nucl. Sci., pt. 2, vol. 46, no. 3, pp. 558-564, Jun. 1999.

[11] M. Khodaverdi, F. Pauly, and S. Weber, "Preliminary studies of a MicroCT for a combined small animal PET/CT scanner," in Proc. IEEE Nuclear Science Symp. and Medical Imaging Conf., San Diego, CA, Nov. 4-10, 2001, pp. 1605-1606.

[12] P. L. Chow, A. L. Goertzen, and F. Berger, "Monte Carlo model for estimation of dose delivered to small animals during 3D high resolution X-ray computed tomography," in Proc. IEEE Nuclear Science Symp. and Medical Imaging Conf., San Diego, CA, Nov. 4-10, 2001, pp. 1678-1681. 